\title{
Design and Implementation of Vehicles Monitoring System Based on Android Mobile Terminals
}

\author{
Nuan Sui 1,a, Chunhai Zhang ${ }^{1, b}$, Hao Liu' ${ }^{1, c}$, Yanxiu Sheng ${ }^{1, d}$, Xi Wang ${ }^{2, e}$ and \\ Zhiqiang Wei ${ }^{1, f}$ \\ ${ }^{1}$ College of Information Science and Engineering, Ocean University of China, Qingdao, China \\ ${ }^{2}$ Central Research Institute of Haier Group, Qingdao, China \\ asuinuan5125@126.com,bchai@ouc.edu.cn, cliu.hao@msn.com, ${ }^{\mathrm{a}}$ shengyanxiu@ouc.edu.cn, \\ ewangxi@haier.com, 'weizhiqiang@ouc.edu.cn
}

Keywords: android; vehicles monitoring system; real-time monitoring; historical trace

\begin{abstract}
With the popularity of android smartphone and the increasing number of the vehicles, the problems of obtaining real-time vehicle status information and historical trace information have attracted considerable attention. For these two problems, the vehicle monitoring system client running on android smartphone was designed and implemented, which utilizes the internet - enabled smartphone and GPS position information. This friendly and strong system offers features such as login capability, real-time monitoring capability and the ability to query trace. The system has been realized on the Android mobile terminals and performs well.
\end{abstract}

\section{Introduction}

With the popularity of the vehicles, a series of problems, such as the traffic congestion and traffic accident, have occurred with the convenience. These problems have affected the healthy and harmonious development of the personality, enterprise and society to a certain extent. Hence, special customization and expensive vehicle terminal equipment [1] rapidly occupies the market.

Under such circumstance, a vehicle monitoring system based on Android mobile devices [2] is proposed in this paper. It shows three significant characteristics: low cost, the system only requires a common android smartphone. Extensibility, the whole system is easy to extend for its modularization design. Real-time, show the vehicle physical position in real-time on the Baidu Map.

\section{The Design of the System}

The Physics Model of the System [3]. From a global perspective, the physics model of the system contains three parts: database, application server and android mobile terminal. The database is designed to store and manage all data used in the system such as username, password and GPS information. The application server, which is similar to a transfer station, selects database according to the client's requirement, and then, transfers results to client through the Internet. The client receives data from server to analysis, handle and finally display results.

The Logical Model of the System [4]. The logical model of the system contains three levels, namely the data layer, the business layer and the application layer. In the main, the application layer depends on the support offered the business layer and the data layer to show user's location, vehicle's location and historical trace of the vehicle. Fig. 1 shows the logical model of the system. 

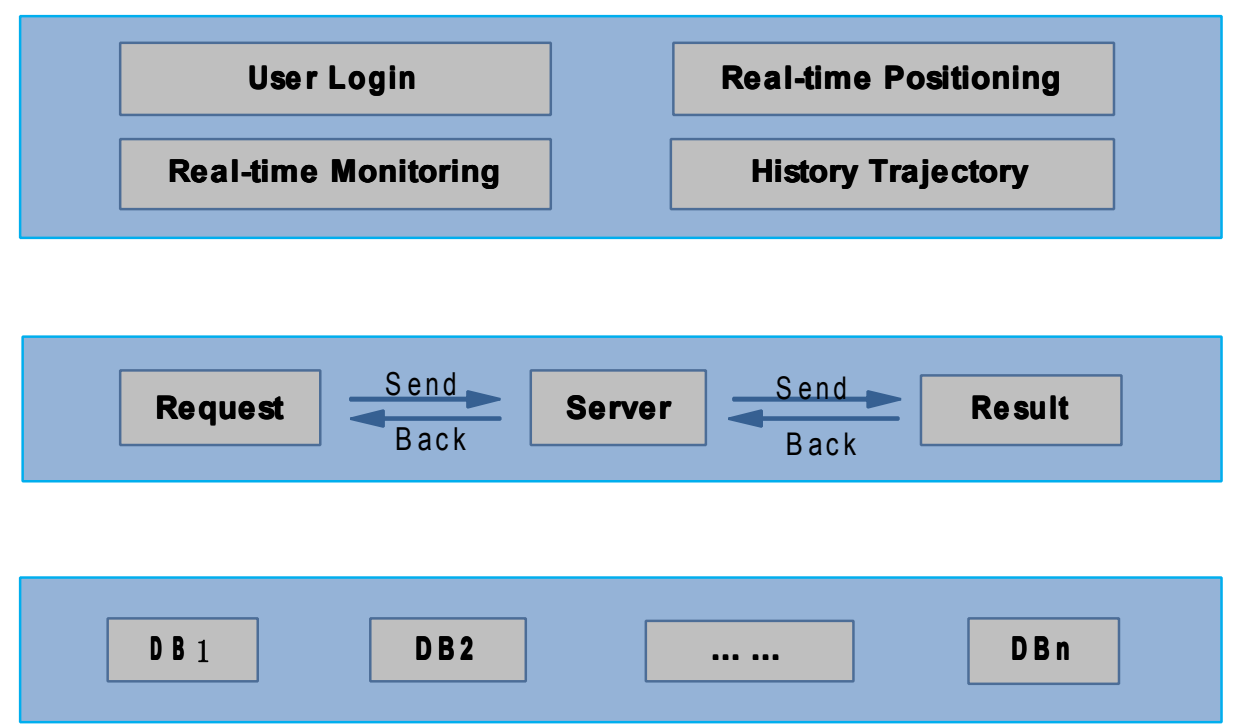

Fig. 1. The logical model of the system

\section{Application \\ Layer}

\section{Business \\ Layer}

\section{Data \\ Layer}

The data layer, which provides data support for the system, is the core component of the system. It stores the physical data files and all the data used in the system.

The business layer [5] mainly offers personalized and intelligent support to the system. Its main benefits are extendable ability and to facilities the optimization and upgrading of the business.

The application layer, which is mainly for the displaying of the system and help the user complete the operation of the system, contains three modules at present. They are login module, real-time monitor module and historical trace module.

\section{Key Techniques}

The Coordinate Transformation [6]. In order to let users more conveniently examine the oneself and target vehicle's relative position on the Baidu Map, an efficient and accurate calculation method based on the cartesian coordinate system and tangent function in mathematics is proposed.

Its theoretical foundation can as thus: the angle(counterclockwise rotation) between the positive $\mathrm{X}$ axis(or the translation positive $\mathrm{X}$ axis) and the ray(A is endpoint) attached any two points ranges from $0^{0}$ to $360^{\circ}$ in the general cartesian coordinate system, which is shown in Fig. 2. According to coordinate inverse formula and quadrant angle of the line $\mathrm{AB} \theta=\operatorname{arc} \tan \left(\Delta \mathrm{y}_{\mathrm{AB}} / \Delta \mathrm{x}_{\mathrm{AB}}\right)\left(\right.$ if $\Delta \mathrm{x}_{\mathrm{AB}}=0$, $\tan \theta=\infty, \theta=90^{\circ}$ or $180^{\circ}$ ), namely $\mathrm{d}=\theta^{*} 180^{\circ} / \Pi$, the calculating results of the angle are shown in Table 1.

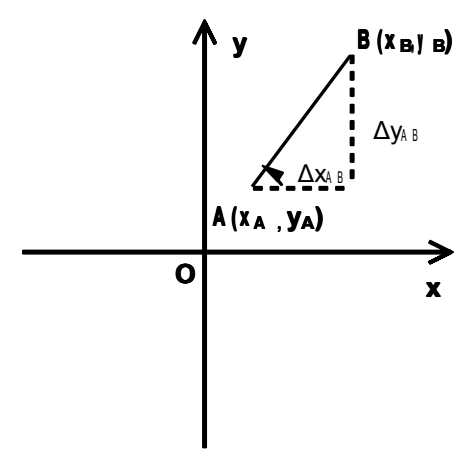

Fig. 2. Coordinates: $\Delta \mathrm{x}_{\mathrm{AB}}=\mathrm{x}_{\mathrm{B}}-\mathrm{x}_{\mathrm{A}}, \Delta \mathrm{y}_{\mathrm{AB}}=\mathrm{y}_{\mathrm{B}}-\mathrm{y}_{\mathrm{A}}$ 
Table 1. The calculation table of the angle

\begin{tabular}{|c|c|c|}
\hline$\Delta \mathrm{x}_{\mathrm{AB}}$ & $\Delta \mathrm{y}_{\mathrm{AB}}$ & The degree of the angle[degree] \\
\hline+ & + & $\mathrm{d}$ \\
\hline- & + & $\mathrm{d}+180$ \\
\hline- & - & $\mathrm{d}+180$ \\
\hline+ & - & $\mathrm{d}+360$ \\
\hline
\end{tabular}

The specific calculation method can be divided into four processes, and the details of each process are described as follows:

The coordinate establishment. For ease of calculation, the new cartesian coordinate system whose coordinate origin is $\mathrm{O} 1$ - the center of the screen is created, as the Fig. 3 illustrates. It is indicated by $(\mathrm{x}, \mathrm{y})$ and also in pixels.

The GPS coordinates are converted into the screen coordinates by android utility classes [7].

Making assumptions. First, lower right area is named the first quadrant, the other three areas in clockwise are separately named the second quadrant, the third quadrant and the forth quadrant. Second, the direction of rotation is clockwise.

The angle calculation. According to the above theory, the angle between the positive $\mathrm{X}$ axis and the ray(the center of the screen is endpoint) attached the center of the screen and any one points located in the cartesian coordinate system is shown as Table 2.

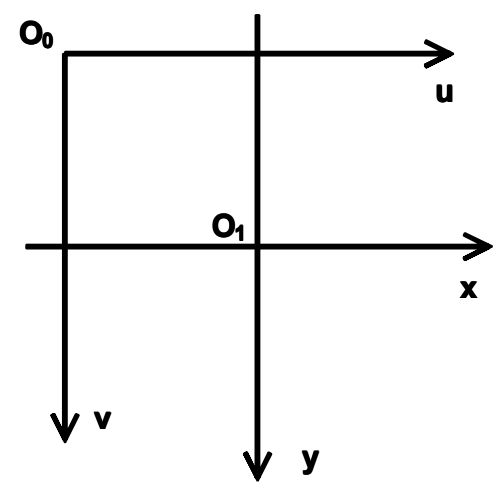

Fig. 3. Coordinates: In the android screen coordinates, the coordinate origin is $\mathrm{O}_{0}$ - the upper left corner of the screen, the positive $\mathrm{X}$ axis is defined from left to right, the $\mathrm{Y}$ positive axis is defined from up to down. It is indicated by $(\mathrm{u}, \mathrm{v})$ and in pixels.

Table 1. The calculation table of the angle

\begin{tabular}{|c|c|}
\hline The location of point & The degree of the angle[degree] \\
\hline the positive X axis & 0 \\
\hline the first quadrant & $\mathrm{d}$ \\
\hline
\end{tabular}




\begin{tabular}{|c|c|}
\hline the positive Y axis & 90 \\
\hline the second quadrant & $\mathrm{d}+180$ \\
\hline the negative X axis & 180 \\
\hline the third quadrant & $\mathrm{d}+180$ \\
\hline the negative Y axis & 270 \\
\hline the fourth quadrant & $\mathrm{d}+360$ \\
\hline
\end{tabular}

Improved MD5 Encryption Algorithm [8]. In order to ensure the security of the password, SharedPreferences is widely used. However, password can be easily got by FileBrower software so that the password is easily leaked.

So, MD5 encryption algorithm is widely used. It is the hash function designed by Ron Rivest as a strengthened version of MD4. The brief description of MD5 encryption algorithm is as follows: MD5 processes input information in 512-bit message block. And every group is divided into 16 32-bit sub-message blocks. After a series of processing, the algorithm's output is composed of 4 32-bit message blocks, and then, 4 32-bit message blocks cascaded generate a 128-bit hash value. MD5 encryption algorithm has two important characteristics. First, it is anti-collision which consists of the different message with two different sets of initial values. Second, the result of the same input is forever the same one.

However, there are many methods cracking MD5 encryption algorithm. "Running dictionary" is widely used. For this, there are two solutions in general. One method is to strengthen the password complexity such as longer length and multiple symbol combinations. Another method is to improve MD5 encryption algorithm [9]. Though the former method is simple, its security remains relatively weak. The second method is used in this paper.

The specific process is as follows:

Encryption. The MD5 encryption algorithm is utilized to generate the ciphertext of original password, namely $\mathrm{h}=\mathrm{H}(\mathrm{M})$.

Division. The ciphertext is divided into two equal blocks, namely $\mathrm{h} 1+\mathrm{h} 2=\mathrm{h}$.

Combination. The original password is placed between the two blocks changed the order, namely $\mathrm{M}^{\prime}=\mathrm{h} 2+\mathrm{M}+\mathrm{h} 1$.

Re-encryption. The MD5 encryption algorithm is utilized again to generate the ciphertext of M', namely h'=H(M').

Ciphertext. h' is the final ciphertext.

Fig. 4 shows the process of improved MD5 encryption algorithm.

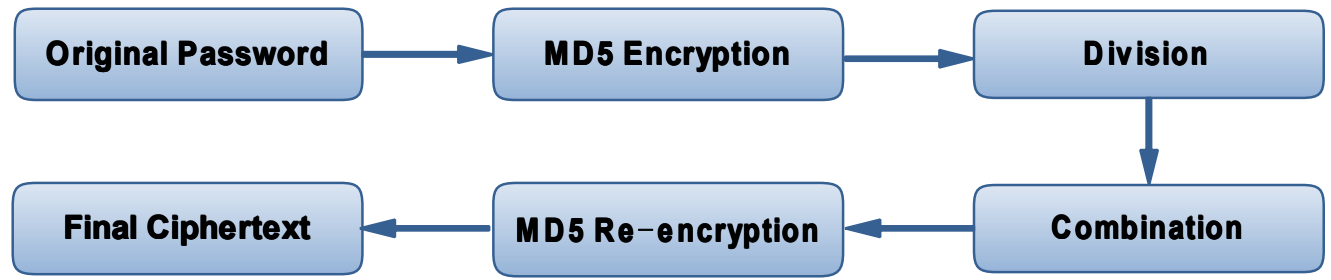

Fig. 4. The process of improved MD5 encryption algorithm

\section{Conclusion}

In this paper, the basic architecture and key technologies are specified, which are utilized in 
vehicles monitoring system and discussed methods that make the system effective and friendly to users. This system has run on android smartphone and realized real-time monitoring of vehicles without the expensive terminal devices.

The next step is going to further optimize and expand system's functions. On one hand, the data flow will be reduced. On the other hand, the useful functions such as collecting and saving the evidence will be added.

\section{References}

[1] Song Qingkun. Design and Implementation of GPS Vehicle Monitoring System Terminal [D]. Harbin: Harbin University of Science and Technology, 2009.

[2] Cao Gaofeng, Wu Jianping. The Design of Logistic Vehicle Scheduling Platform Based on Android Intelligent Terminal[J]. Computer Science and Application, 2012, 2(3): 147-151.

[3] B. B. Chen and M. C. Chan, "MobTorrent: a framework for mobile Internet access from vehicles," in Proc. IEEE INFOCOM'09, pp. 1404-1412, Apr. 2009.

[4] Yao Yuyu, Liu Weiguo. Android Architecture and Application Development Research[J]. Computer System and Application, 2008, 17(11): 110-112.

[5] Geng Dongjiu, Suo Yue, Chen Yu, et al. The Remote Access and Control System Based on Android Mobile[J]. Computer Application, 2011, 31(2): 559-560.

[6] Android Programming-GPS Positioning, Android Development Forum, DevDiv Mobile Development Community[EB/OL].[2011-05-21]. http://www.devdiv.com/thread-33143-1-21.html.

[7] Cao Ke, Wu Yue. Application of Assisted-Global Positioning System on 3G Terminal[J]. Computer Technology and Development, 2006, 16(12):139-141.

[8] Xu Maozhi, You Lin. Information Security and Cryptography[M]. Beijing: Tsinghua University Press, 2007.121 127.

[9] Guo Shanqiong. Research and Implementation of MD5 Data Encryption Algorithm[D]. Wuhan: Wuhan University of Technology, 2007:31-36.

\section{Acknowledgment}

We acknowledge Ph.D Hao Liu, Wei Cheng for their instructive suggestions and valuable advice. Supported by the National Basic Research Program of China (Grant No. 2006039) and Technology Development and Cooperation (Grant No. 20120465). 